\title{
Association between a syndemic of psychosocial problems and unprotected anal intercourse among men who have sex with men in Shanghai, China
}

Ying Wang ${ }^{1}$, Zezhou Wang ${ }^{1}$, Mengmeng Jia ${ }^{1}$, Ajuan Liang ${ }^{2}$, Dong Yuan ${ }^{3}$, Zhe Sun ${ }^{1}$, Feng Gan ${ }^{4}$, Yichen Wang ${ }^{5}$, Yong Cai ${ }^{*}$ and Zhiruo Zhang ${ }^{1 *}$

\begin{abstract}
Background: Previous studies have discussed the overlapping and reinforcing effects (defined as a syndemic) of psychosocial problems on high-risk sexual behaviors among men who have sex with men (MSM). The present study aimed to apply the syndemic theory to verify the reinforcing effects of psychosocial problems on unprotected anal intercourse (UAI) among MSM in Shanghai, and determine if other important psychosocial factors fit into the syndemic theory.

Methods: Data were collected from 547 MSM in Shanghai, China, through face-to-face interviews. The measures for psychosocial problems included the Rosenberg Self-Esteem Scale; the Generalized Anxiety Disorder-7; the Center for Epidemiological Studies Depression Scale; the University of California, Los Angeles Loneliness Scale; and the Sexual Compulsivity Scale. We used multivariate analysis and binary logistic regression to investigate the associations between psychosocial problems and high-risk sexual behaviors.

Results: The prevalence of UAI among MSM in the past 6 months was $54.5 \%$. Education (graduate from college vs. high school) served as a protective factor against UAI (OR 0.59, 95\% Cl: 0.38-0.94). There was a high prevalence of psychosocial problems, and at least one-third of participants reported experiencing more than two psychosocial symptoms. Of these psychosocial factors that we investigated, lower self-esteem was associated with UAI in both univariate and multivariate regression model $(P=0.009)$. Result suggests that overlapping and reinforcing effects of psychosocial problems may increase high risk sexual behaviors among MSM in Shanghai, China (OR 1.65, 95\% Cl: $1.09-2.50 ; P=0.018)$.

Conclusions: We found further evidence for a syndemic of psychosocial problems among MSM in Shanghai, China. This syndemic may also increase high risk sexual behaviors among MSM. Most HIV prevention interventions are focused on behavior change and only have moderate effects; our findings suggest that a shift from behavior-focused interventions to a more comprehensive strategy that addresses psychosocial factors may be necessary.
\end{abstract}

Keywords: MSM, Psychosocial problems, Syndemic

\footnotetext{
* Correspondence: caiyong202028@hotmail.com; zhangzhiruo@sjtu.edu.cn ${ }^{1}$ School of Public Health, affiliated with School of Medicine, Shanghai Jiao Tong University, Shanghai 200025, China

Full list of author information is available at the end of the article
} 


\section{Background}

The group of men who have sex with men (MSM) accounts for the largest number of new HIV infections [1]. The HIV infection rate in the Asian MSM population has dramatically increased in recent years, and unprotected anal intercourse (UAI) and multiple sexual partners (MSP) are considered to be the leading risk factors for HIV infection among MSM [2-5]. This is also the case in China, and as MSM who have UAI with men may also have unprotected sex with women, there is a risk of spreading HIV to the general population [6]. There are an estimated 10 to 25 million MSM in China; estimates of the HIV prevalence due to high risk sexual behaviors (especially UAI) in the MSM population range from 6.5 to $7.2 \%[6-8]$. When it comes to high-risk sexual behaviors among MSM, it mainly means UAI and MSP. Of those living with HIV/AIDS in China, an estimated $27.2 \%$ were infected via unprotected sexual intercourse among MSM $[9,10]$.

The MSM population may not be accepted by most Chinese families. The pressure of being a minority group discriminated by mainstream society may cause psychosocial problems, such as depression and anxiety $[1,2,11,12]$. Estimates of the prevalence of depression in the MSM population ranged from 29.2 to $63.9 \%$, which were mostly due to social acceptance for homosexuality and other factors such as HIV-related stigma, whereas in the general population the prevalence was 5.3 to $23 \%$ [13]. Other psychosocial problems such as anxiety have also been suggested to be more prevalent among MSM than in the general population [14].

Some studies have noted that the high-risk sexual behaviors of MSM such as UAI were highly correlated with their psychosocial problems, and these psychosocial problems might contribute to HIV vulnerability $[9,15,16]$. Serious psychosocial problems may also lead to high-risk sexual behaviors. Research on MSM in India showed that MSM with depressive symptoms tended to participate in UAI [17]. Results from the article written by De Santis JP and Wilson PA indicated that higher levels of depressive symptoms had a statistically significant relationship to higher levels of high-risk sexual behaviors such as UAI. They suggested that increases in depression among sexually active MSM may result in externalizing symptoms such as sensation-seeking and the use of sex to avoid or alleviate negative affect $[18,19]$. Similar conclusions were drawn in studies with MSM in Spain, rural areas, and in older adults [18, 20, 21]. Psychosocial problems may also be obstacles that prevent the MSM population from using health services. Research indicates that MSM with psychosocial problems usually do not visit a doctor even if when they are unwell $[6,22]$. Similarly, researchers in China concluded that psychosocial problems may increase the vulnerability to HIV among MSM $[9,23]$. It is important to understand the effect of co-occurring psychosocial problems on behavior as well. Psychosocial problems may interact with each other (syndemic) and increase the risk of HIV infection [2, 17, 24-26]. A syndemic is defined as synergistically interacting health problems coming together and producing additional disease in a population; the various factors may have an additive effect and intensify each other [16, 27]. This was first found by Stall when he surveyed MSM in four major American cities [26]. And the United States Center for Disease Control and Prevention (CDC) defined syndemic as more than two factors interacting with each other, and potentially causing an extra burden on people [28, 29]. A number of studies have shown that psychosocial problems were syndemic factors which might lead to high-risk sexual behaviors among MSM [6, 30, 31]. High-risk sexual behaviors have also been found to be highly correlated with the number of psychosocial problems [15-17, 32]. Another survey found that the more frequently MSM reported participating in UAI, the more psychosocial problems there were [33].

Most research on MSM in China has focused on demographic characteristics, sexual behaviors, and HIV infection [34, 35]; only a small number of studies have explored the psychosocial context [36, 37], and even fewer have considered the interacting and overlapping effects of psychosocial factors [11, 12]. In the present study, we investigated the prevalence of psychosocial problems in MSM in Shanghai, to determine if a syndemic of psychosocial problems could increase high-risk sexual behaviors and HIV infection in this population. Previous studies on the syndemic theory outline a framework consisting of several essential factors: drug use, depression, childhood abuse, and intimate partner violence; all of which were verified in most studies [1, 17, 21, 26, 38]. Other studies have shown that factors such as smoking, heavy alcohol use, anxiety, sexual compulsivity, and suicidal ideation may also fit into the syndemic theory [12, 15, 23, 39]. Selfesteem and loneliness may also be important psychosocial factors for MSM, yet studies about these factors were disproportionately rare $[21,25,40,41]$. Self-esteem is an important predictor of depressive symptoms, and people with lower self-esteem may experience higher stigma and be more depressed [42]. An association between homophobic stigma and risk-taking behaviors has been found [43]. Another study showed that as loneliness increased, condom use among MSM decreased [44]. Therefore, we chose self-esteem and loneliness as psychosocial factors in addition to anxiety, depression, and sexual compulsivity. We hypothesized that these five co-occurring factors (selfesteem, anxiety, depression, loneliness, and sexual compulsivity) may interact synergistically, reinforce each other, and finally impose an extra burden on MSM in Shanghai, 
China. Our findings may provide evidence in China for the syndemic theory, and show that different psychosocial factors may interact synergistically to increase HIV risk in the MSM population. If this is the case, attention should be directed to designing tailored, preventive strategies and holistic public health policies targeted to this population subgroup.

\section{Methods}

\section{Study setting}

Shanghai is the economic center of China, and with its fast-growing economy and tolerance for subculture, it attracts MSM from all over the country. Our research group has a long-term cooperative relationship with the Shanghai Center for Disease Control and Prevention, the Shanghai Dermatology Hospital, and the Shanghai Youth AIDS Health Promotion Centre (a non-governmental organization). This cooperative relationship enabled us to conduct our study with MSM in Shanghai. Our target population was men who had sex with men in the past 6 months, regardless of how they identified themselves (e.g., bisexual, homosexual, or heterosexual).

\section{Study and sampling procedure}

The inclusion criteria were men who had sex with men in the past 6 months and were aged over 16 years. As they experience discrimination from mainstream society, most MSM tend to hide their sexual orientation. Therefore, it would be difficult to identify MSM and use a random trial method to conduct our survey. Instead, we used a snow-balling method to recruit eligible participants, and this proved to bean efficient way to approach a minority population. First, with help from the Shanghai Youth AIDS Health Promotion Centre, we located some individuals who met the inclusion criteria and invited them to complete the questionnaire. We then asked these initial participants to introduce other men eligible to take part in the survey. We repeated this procedure until we obtained the necessary sample size.

A review of the literature highlighted that the two most important risk indicators for HIV infection among MSM were UAI and MSP. We selected UAI as the observed variable. The reported prevalence of UAI among MSM varied from 41 to $78 \%$ (average of $50 \%$ ) [9, 32, 38]. Assuming a UAI prevalence of $50 \%$, an $\alpha$ of 0.05 and a relative sampling error of $0.1 \mathrm{P}$, we calculated the necessary sample size as 400 . We increased this by $50 \%$ to compensate for any sampling error. Given a target response rate of $95 \%$, the final required sample size was 463 .

In total, 567 MSM agreed to participate and 547 participants completed the questionnaire (response rate of 96\%). Data were collected in face-to-face interviews. Each interview lasted about $30 \mathrm{~min}$, and participants were paid 100
CNY (16.34 USD) before the interview as compensation for travel expenses.

\section{Statistical analysis}

Data were double-entered using Epidata 3.0. Data were analyzed with the Statistical Program for Social Sciences version 20.0 (IBM SPSS Statistics for Windows, Version 20.0. Armonk, NY: IBM Corp.). Descriptive statistics, such as means, standard deviations (SD), frequencies, and percentages were used to examine the sociodemographic characteristics of MSM in Shanghai.

We analyzed data in five steps. First, we described the demographic characteristics and evaluated their association with UAI by univariate logistic regression. Second, we analyzed psychosocial factors and tested their association with UAI by univariate logistic regression after adjusting for statistically significant demographic variables. Third, we used a forward stepwise multivariate logistic regression to evaluate risk psychosocial factors after adjusting for significant demographic variables. Fourth, we conducted binary logistic regression to test the syndemic effects between all psychosocial factors and UAI after adjusting for significant demographic variables. Finally, we also tested the syndemic effects after excluding significant psychosocial factors which were verified in step three. All the significance level was set at 0.05.

\section{Ethical considerations}

We obtained approval for the present study from the Ethics Committee of the School of Public Health Shanghai Jiao Tong University. Background information about the survey was given to all participants, after which they were given written informed consent forms, which set out the goal and procedure of the study, as well as the potential risks. Written informed consent was obtained from all participants before the study began. During the recruitment and interview procedure, participants were free to ask any questions and to withdraw if they did not wish to continue.

\section{Measures \\ Background characteristics}

Socio-demographic characteristics including age, educational level, marital status, monthly income, residential status, self-report sexual orientation, and self-reported HIV status were collected.

\section{Psychosocial problems}

Psychosocial problems including self-esteem, anxiety disorder, depression, loneliness, and sexual compulsivity were measured using standard scales.

\section{Rosenberg self-esteem scale}

Self-esteem is correlated with many other indicators of psychosocial health problems, and is considered to core 
measurement of psychosocial health problems [45]. The Rosenberg Self-Esteem Scale is a reliable and valid tool to assess self-esteem and is widely used in scientific research [46]. It consists of 10 Likert-type items, for example, "I feel that I am a person of worth, at least I am equal to others" [47]. Responses to all items are on a 4-point scale, ranging from strongly agree to strongly disagree. For items 1, 2, 4, 6, and 7, "strongly agree" is scored 3, "agree" is scored 2, "disagree" is scored 1 , and "strongly disagree" is scored 0 . Items $3,5,8,9$, and 10 are reversed ("strongly agree" is scored 0 and "strongly disagree" is scored 3 ). The score range was 0 to 30 (Cronbach's alpha coefficient 0.839 ). Scores between 15 and 25 are considered to be within the normal range, and scores below 15 suggest low self-esteem [46].

\section{Generalized anxiety disorder-7 (GAD-7)}

Standardized rating scales such as the GAD-7are used to assess severity of symptoms of generalized anxiety disorder. The GAD-7 has also been used to diagnose and assess anxiety symptoms [48]. It comprises 7 items describing symptoms that participants may have experienced in the previous2 weeks (e.g., I feel nervous and upset). Responses to all items are on a 4-point scale $(0=$ totally none to $4=$ almost every day). Total scores range from 0 to 28 (Cronbach's alpha coefficient 0.922 ), with scores over 10 suggesting the presence of anxiety disorder [48].

\section{Center for epidemiological studies depression scale (CESD)} Depressive symptoms were assessed with the CESD. Participants are asked 20 questions to assess the depressive symptoms they experienced in the previous 1 week (e.g., I feel sad.). Each item is scored from 0 to $3(0=$ rarely or none of the time, $1=$ some or little of the time, $2=$ moderately or much of the time, and $3=$ most or almost all the time). Items $4,8,12$, and 16 are reversed in valence. Scores for each item are summed to provide a total score. The scores ranged from 0 to 60 (Cronbach's alpha coefficient 0.891). Higher scores indicate more severe depressive symptoms. Scores over 16 were used to define major depressive symptoms.

\section{University of California, Los Angeles Loneliness Scale (UCLA Loneliness Scale)}

Loneliness symptoms were assessed using the 8-item UCLA Loneliness Scale (e.g., "I feel isolated"). Responses are on a 4-point Likert scale ranging from 0 to 3 . Items 3 and 6 were reversed in valence (Cronbach's alpha coefficient 0.829$)$. Scores over 18 suggest loneliness symptoms.

\section{Sexual compulsivity scale}

Sexual compulsivity was assessed with 10 questions or statements (e.g., "I think of sex when I am working"). Item 1 is reversed in valence (Cronbach's alpha coefficient 0.860). Scores over 26 indicate sexual compulsivity symptoms.

\section{Syndemic of psychosocial factors}

If more than two psychosocial problems occurred at the same time in an individual participant, we assumed there was a syndemic phenomenon. A syndemic variable was created by counting the number of psychosocial factors.

\section{Unprotected anal intercourse}

Participants were asked questions about condom use when having anal sex with male sex partners in the past 6 months. We used a Likert scale to measure condom use, with response options ranging from 1 (never) to 5 (always). Men who responded with a number other than 5 (always) were considered to have participated in unprotected sex when having anal sex.

\section{Results}

\section{Participants' characteristics}

In total, 547 participants completed the questionnaire. Table 1 presents the participants' demographic characteristics. The age of participants ranged from 17.33 to 65.33 years (mean $=30.50$ years; $\mathrm{SD}=8.84$ years $)$. Those aged 25 to 40 years accounted for $61.6 \%$ of participants. The majority $(71.3 \%)$ of participants went to college, indicating that most participants had a background of higher education; $79.3 \%$ were single and $15.0 \%$ were married to a woman. Around $24.3 \%$ of participants earned less than 3000 RMB per month (approximately 488 USD), and $73.1 \%$ came from outside Shanghai. Of the participants, $71.3 \%$ identified themselves as homosexual and $6.8 \%$ of participants self-reported being HIV positive. In addition, the prevalence of UAI in the past 6 months was $54.5 \%$.

\section{Psychosocial factors}

Table 2 describes the prevalence of participants' individual psychosocial factors. Around 10.6\% of participants reported having low self-esteem, $12.2 \%$ reported experiencing anxiety, 52.1\% reported feeling depressed, and 24.2\% reported feeling lonely. The prevalence of sexual compulsivity was 20.3\%. Approximately 35.5\% reported having more than two psychosocial problems, which were considered syndemic of the five psychosocial factors.

Socio-demographic characteristics and associations with UAI We used a univariate logistic regression to analyze the association between socio-demographic characteristics and UAI (Table 3). Those with a high educational background (graduate from college) were less likely to participate in UAI, and those with a poor educational background were more likely to do so (OR 0.59, 95\% CI: 0.38-0.94). Participants with a monthly income between 
Table 1 Socio-demographic characteristics of men who have sex with men in Shanghai, China $(n=547)$

\begin{tabular}{|c|c|c|}
\hline Characteristics & $n$ & $\%$ \\
\hline Age (years) & $(17.33-65.33)$ & \\
\hline$<25$ & 148 & 27.1 \\
\hline $25-40$ & 337 & 61.6 \\
\hline$>40$ & 62 & 11.3 \\
\hline \multicolumn{3}{|l|}{ Education } \\
\hline High school & 157 & 28.7 \\
\hline College & 390 & 71.3 \\
\hline \multicolumn{3}{|l|}{ Marital Status } \\
\hline Single & 434 & 79.3 \\
\hline Married & 82 & 15.0 \\
\hline Divorced or Widowed & 31 & 5.7 \\
\hline \multicolumn{3}{|l|}{ Monthly income (CNYa) } \\
\hline$<3000$ & 133 & 24.3 \\
\hline $3000-6000$ & 211 & 38.6 \\
\hline$>6000$ & 203 & 37.1 \\
\hline \multicolumn{3}{|l|}{ Residential status } \\
\hline Local inhabitant & 147 & 26.9 \\
\hline Non-local inhabitant & 400 & 73.1 \\
\hline \multicolumn{3}{|c|}{ Self-report Sexual orientation } \\
\hline Not homosexual & 157 & 28.7 \\
\hline Homosexual & 390 & 71.3 \\
\hline \multicolumn{3}{|l|}{ HIV Positive } \\
\hline Yes & 24 & 4.4 \\
\hline No & 523 & 95.6 \\
\hline \multicolumn{3}{|c|}{ Unprotected anal intercourse (UAI) } \\
\hline No & 249 & 45.5 \\
\hline Yes & 298 & 54.5 \\
\hline
\end{tabular}

${ }^{a}$ CNY 3000 equivalent to 480 USD; 6000 equivalent to 960 USD

3000 and 6000 were more likely to engage in UAI when compared with those with a monthly income less than 3000 (OR 1.90, 95\% CI: 1.20-3.01).

\section{Associations between psychosocial factors and UAI}

After controlling for demographic factors such as education and monthly income, we used a binary logistic regression model to analyze the relationships between psychosocial factors and UAI. Table 4 shows that after controlling for demographic factors. Self-esteem was significant in univariate logistic regression. We conducted a multivariate logistic regression to evaluate the multiple psychosocial problems (including self-esteem) with UAI among MSM. The result showed that selfesteem remained significant $(P=0.009)$. However, most $p$ values of other four psychosocial factors were above 0.05 ,
Table 2 Psychosocial factors of MSM in Shanghai, China $(n=547)$

\begin{tabular}{lll}
\hline Factors & $N$ & $\%$ \\
\hline Rosenberg Self-Esteem Scale & & \\
High level (Score $\geq 15)$ & 489 & 89.4 \\
Low level (Score <15) & 58 & 10.6 \\
GAD Anxiety & & 87.8 \\
Low level (Score <10) & 480 & 12.2 \\
High level (Score $\geq 10)$ & 67 & \\
CESD & & 47.9 \\
Low level (Score <16) & 262 & 52.1 \\
High level (Score $\geq 16)$ & 285 & 75.8 \\
UCLA Loneliness Scale & & 24.2 \\
Low level (Score $\leq 18)$ & 414 & \\
High level (Score >18) & 132 & 79.7 \\
Sexual compulsivity & & 20.3 \\
Low level (Score $\leq 26)$ & 435 & 35.5 \\
High level (Score >26) & 111 & \\
Syndemic of five factors & & \\
No & 353 & \\
Yes & 194 & \\
\hline
\end{tabular}

indicating that the association between UAI and single psychosocial factors was ambiguous.

If two or more than two psychosocial factors (syndemic of five psychosocial factors) occurred at the same time for a single participant, they were more likely to report engaging in UAI in the past 6 months (OR 1.65, $95 \%$ CI: $1.09-2.50 ; P=0.018$ ). As self-esteem was significant in both univariate and multivariate regression model, we tried to exclude self-esteem from the model to avoid its effects, and use other four psychosocial factors to verify the overlapping and reinforcing effects on UAI (syndemic of four psychosocial factors). Results still showed positive associations between these four syndemic factors and UAI among MSM, Shanghai, China (OR 1.52, 95\% CI: 1.06-2.20) (Table 5).

\section{Discussion}

We found that more than half of the participants $(54.5 \%)$ reported having had UAI in the past 6 months. This indicates that a high number of MSM in Shanghai have engaged in high-risk sexual behaviors, particularly as the prevalence of HIV was $6.8 \%$. Our finding suggests an urgent need to promote safe sex among MSM to prevent HIV spread to the general population. This was consistent with findings from other studies $[6,9]$.

We found that a background of higher education was a protective factor against UAI. It may be that those with a better education understand more about HIV prevention. The association between monthly income and UAI was more complicated. Our findings indicated that to a 
Table 3 Associations between socio-demographic characteristics and unprotected anal intercourse among MSM in Shanghai, China

\begin{tabular}{|c|c|c|c|}
\hline Characteristics & $N$ & $\%$ & ORu $(95 \% \mathrm{Cl})$ \\
\hline \multicolumn{4}{|l|}{ Age (years) } \\
\hline$<25$ & 76 & 51.4 & 1 \\
\hline $25-40$ & 187 & 55.5 & $1.06(0.69-1.63)$ \\
\hline$>40$ & 35 & 56.5 & $1.10(0.54-2.21)$ \\
\hline \multicolumn{4}{|l|}{ Education } \\
\hline High school & 99 & 63.1 & 1 \\
\hline College & 199 & 51.0 & $0.59(0.38-0.94)^{*}$ \\
\hline \multicolumn{4}{|l|}{ Marital Status } \\
\hline Single & 233 & 53.7 & 1 \\
\hline Married & 48 & 58.5 & $0.86(0.50-1.50)$ \\
\hline Divorced or Widowed & 17 & 54.8 & $0.76(0.34-1.70)$ \\
\hline \multicolumn{4}{|l|}{ Monthly income (CNY $)$} \\
\hline$<3000$ & 64 & 48.1 & 1 \\
\hline $3000-6000$ & 132 & 62.6 & $1.90(1.20-3.01)^{*}$ \\
\hline$>6000$ & 102 & 50.2 & $1.32(0.79-2.20)$ \\
\hline \multicolumn{4}{|l|}{ Residential status } \\
\hline Local inhabitant & 80 & 54.4 & 1 \\
\hline Non-local inhabitant & 218 & 54.5 & $0.91(0.60-1.37)$ \\
\hline \multicolumn{4}{|c|}{ Self-reported Sexual orientation } \\
\hline Not homosexual & 91 & 58.0 & 1 \\
\hline Homosexual & 207 & 53.1 & $0.86(0.58-1.28)$ \\
\hline
\end{tabular}

ORu odds ratio obtained from forward stepwise univariate logistic regression ${ }^{a}$ CNY 3000 equivalent to 480 USD; 6000 equivalent to 960 USD; ${ }^{*} p<0.05$ certain level, the prevalence of UAI increased as monthly income increased. However, this pattern of behavior changed when monthly income exceeded a certain amount (6000RMB).

Most research on MSM in China has focused on highrisk sexual behaviors. There was a paucity of literature exploring psychosocial factors and their relationship to high-risk sexual behaviors. Our findings indicated a high prevalence of psychosocial problems among MSM in Shanghai, China. More than half of the participants (52.1\%) suffered from depression and 35.5\% had been diagnosed with at least two symptoms of psychosocial problems. Of these five psychosocial factors, lower selfesteem was associated with UAI in both univariate and multivariate regression model, while other factors were insignificant. Although the occurrence of a single psychosocial factor was not necessarily associated with UAI, there was a greater chance that he might engage in UAI (OR 1.65, 95\% CI: $1.09-2.50 ; P=0.018$ ) if a participant suffered from more than two psychosocial factors. Even if we excluded self-esteem from the model and employed another univariate logistic regression analysis, the syndemic effect of psychosocial problems on UAI was still significant. This supported our hypothesis that the overlapping and reinforcing effect (syndemic) of psychosocial problems might exist in MSM in Shanghai, China. In addition, the co-occurrence of psychosocial factors may fuel high-risk sexual behaviors and HIV infection in MSM in Shanghai, China.

Most intervention strategies targeting this population subgroup focus on behavioral change. These strategies have only had a moderate effect in reducing high-risk

Table 4 Associations between psychosocial factors and UAl among MSM, Shanghai, China $(n=547)$

\begin{tabular}{|c|c|c|c|c|c|c|}
\hline Factors & $n$ & $\%$ & ORu $(95 \% \mathrm{Cl})$ & AOR $(95 \% \mathrm{Cl})$ & ORm $(95 \% \mathrm{Cl})$ & $P$ \\
\hline \multicolumn{7}{|l|}{ Rosenberg Self-esteem } \\
\hline High level (Score $\geq 15$ ) & 257 & 52.6 & 1 & 1 & & \\
\hline Low level (Score <15) & 41 & 70.7 & $2.18(1.20-3.94)^{*}$ & $1.94(1.06-3.56)^{*}$ & $2.19(1.21-3.95)^{* *}$ & 0.009 \\
\hline \multicolumn{7}{|l|}{ GAD Anxiety } \\
\hline Low level (Score <10) & 257 & 53.5 & & & & \\
\hline High level (Score $\geq 10$ ) & 41 & 61.2 & $1.37(0.81-2.31)$ & $1.42(0.83-2.42)$ & - & - \\
\hline \multicolumn{7}{|l|}{ CESD } \\
\hline Low level (Score <16) & 137 & 52.3 & 1 & 1 & & \\
\hline High level (Score $\geq 16$ ) & 161 & 56.5 & $1.19(0.85-1.66)$ & $1.19(0.84-1.67)$ & - & - \\
\hline \multicolumn{7}{|l|}{ UCLA Loneliness } \\
\hline Low level (Score $\leq 18$ ) & 218 & 52.7 & 1 & 1 & & \\
\hline High level (Score >18) & 79 & 59.8 & $1.34(0.90-2.00)$ & $1.25(0.83-1.88)$ & - & - \\
\hline \multicolumn{7}{|l|}{ Sexual compulsivity } \\
\hline Low level (Score $\leq 26$ ) & 228 & 52.4 & 1 & 1 & & \\
\hline High level (Score >26) & 69 & 62.2 & $1.49(0.97-2.29)$ & $1.42(0.92-2.19)$ & - & - \\
\hline
\end{tabular}


Table 5 Associations between syndemic factors and UAl among MSM, Shanghai, China $(n=547)$

\begin{tabular}{llll}
\hline & Number (\%) & MSM who have UAI & AOR (95\% Cl) \\
\hline Have a syndemic of five psychosocial factors & & $177(50.1)$ & 1 \\
$\quad$ No (have no more than 1 psychosocial problem) & $353(64.5)$ & $121(62.4)$ & $1.65(1.09-2.50)^{*}$ \\
Yes (have 2 or more psychosocial problems) & $194(35.5)$ & $191(51.2)$ & 1 \\
Have a syndemic of four psychosocial factors exclude Self-esteem & & $107(61.5)$ & $1.52(1.06-2.20)^{*}$ \\
$\quad$ No (have no more than 1 psychosocial problem) & $373(68.2)$ & $174(31.8)$ & \\
Yes (have 2 or more psychosocial problems)
\end{tabular}
${ }^{*} p<0.05$

behaviors for HIV [1, 49]. As the additive effect of psychosocial problems may reduce the effects of HIV preventive strategies, a holistic framework should be considered for this population. We observed associations between syndemic psychosocial factors, high-risk sexual behaviors, and HIV infection among MSM in Shanghai, China. As 35.5\% of participants had more than two psychosocial problems, and this syndemic may increase high-risk sexual behaviors and HIV infection, a tailored preventive strategy should be implemented that addresses the compounding and amplifying effect between psychosocial factors as well as behavioral interventions.

Our study had several limitations. First, we used a cross-sectional design, and this limited the exploration of influential factors and the inference of causal influences. Second, we used a snow-balling method to identify eligible participants rather than a random trial. All participants were recruited from MSM who attended the Shanghai Youth AIDS Health Promotion Centre to have an HIV test, and therefore excluded those who were unwilling to have an HIV test. Therefore, in a future study we should expand the sample size to cover those who did not want access to a HIV test. Finally, because high-risk sexual behaviors such as UAI are sensitive topics in mainland China and all data were self-reported, participants may not have been completely truthful. Biases in self-reported data were inevitable; however, reliability and validity studies indicated a good test-retest and fit of the results for the measures used.

Despite these limitations, our findings showed that most MSM in Shanghai, China engaged in high-risk sexual behaviors, and suffered from psychosocial problems to some extent. A number of MSM also simultaneously experienced more than two kinds of psychosocial problems, which may have an additive effect. Our results provided evidence to support a holistic approach in intervention strategies targeted to this group that focus on psychosocial factors as well as behavior change. Our goal was not only to promote safe sex, but also to reduce psychosocial problems.

\section{Conclusions}

MSM represents the largest number of the population infected with HIV. This group is also affected by various psychosocial problems. Many studies have suggested strong links between psychosocial burdens, high-risk sexual behaviors, and HIV infection, referred as a syndemic, with a mutually reinforcing (additive or multiplicative) nature. However, to date, little is known about the psychosocial problems in the MSM population in China. Our cross-sectional study investigated the prevalence of psychosocial problems and tested the syndemic theory among MSM in Shanghai, China, and determined the direction and magnitude of the associations between psychosocial factors and high-risk behaviors among MSM. Our findings may provide evidence to inform tailored preventive strategies and public health policies targeting at this population subgroup.

\section{Abbreviations \\ CDC: Center for disease control and prevention; CESD: Center for epidemiological studies depression scale; GAD-7: Generalized anxiety disorder-7; HIV: Human immunodeficiency virus; MSM: Men who have sex with men; MSP: Multiple sexual partners; OR: Odds ratio; SD: Standard deviation; UAl: Unprotected anal intercourse; UCLA Loneliness Scale: University of California, Los Angeles Loneliness Scale}

\section{Acknowledgments}

We are grateful to the study participants for their contribution. We thank the Shanghai Center for Disease Control and Prevention, the Shanghai Dermatology Hospital, and the Shanghai Youth AIDS Health Promotion Centre for helping us to organize the survey. We thank Yi Zhang for her advice for the conception of the manuscript and Dr. Edward C. Mignot for linguistic advice.

\section{Funding}

This study was sponsored by the Shanghai Municipal Education Commission (14YS022), the Shanghai Jiao Tong University School of Medicine (14XJ10007), the Cross-study Research Foundation about Medicine and Engineering of Shanghai Jiao Tong University (YG2014QN23), the National Natural Science Funds of China (71603166, 71673187, 71273174), the Shanghai Pujiang Program (14PJC076), Shanghai Key Discipline Construction Project in Public Health (15GWZK1002), 2016 Shanghai Jiao Tong University School of Public Health -SCDC Research Cooperation Fund and the Social Cognitive and Behavioral Sciences program of Shanghai Jiao Tong University (14JCRY03). The funders had no role in the study design, data collection and analysis, decision to publish, or preparation of the manuscript.

\section{Availability of data and materials}

Because the data have some sensitive information, data will not be shared according to the agreement with the participants. 


\section{Authors' contributions}

YC,YW and other authors discussed, conceived and designed the study. MMJ, AJL, YCW, ZS and ZZW performed the data collection and involved in data analysis. YW and $Y C$ analyzed the data with the suggestions from other authors. FG contributed reagents, materials, analysis tools and the critical revision. YW, YC, DY and ZRZ wrote the paper. YW, YC, ZZW, DY and ZRZ contributed a lot to the revision of the manuscript. All authors read and approved the final manuscript.

\section{Competing interests}

The authors declare that they have no competing interests.

\section{Consent for publication}

Not applicable.

\section{Ethics approval and consent to participate}

Ethical approval was provided by School of Public Health Shanghai Jiao

Tong University. And we got the written consent from the participants.

\section{Author details}

'School of Public Health, affiliated with School of Medicine, Shanghai Jiao Tong University, Shanghai 200025, China. ${ }^{2}$ Renji Hospital, affiliated with School of Medicine Shanghai Jiao Tong University, Shanghai 200127, China. ${ }^{3}$ Shanghai Municipal Center for Disease Control and Prevention, Shanghai 200336, China. ${ }^{4}$ School of Continuing Education, Shanghai Jiao Tong University, Shanghai 200030, China. ${ }^{5}$ Ruijin Hospital, affiliated with School of Medicine, Shanghai Jiao Tong University, Shanghai 200032, China.

\section{Received: 15 April 2016 Accepted: 15 December 2016}

Published online: 07 January 2017

\section{References}

1. Safren SA, Reisner SL, Herrick A, Mimiaga MJ, Stall RD. Mental health and HIV risk in men who have sex with men. J Acquir Immune Defic Syndr. 2010:55 Suppl 2:S74-7.

2. Thomas B, Mimiaga MJ, Kumar S, Swaminathan S, Safren SA, Mayer KH. HIV in Indian MSM: reasons for a concentrated epidemic \& strategies for prevention. Indian J Med Res. 2011;134:920-9.

3. Bai $X$, Luo S, Wang $X$, Yang J, Fan S, Yu M, et al. [Change of risky sexual behaviors among men who have sex with men before and after recent identification of HIV diagnosis]. Zhonghua Liu Xing Bing Xue Za Zhi. 2014:35:489-93.

4. Li HM, Peng RR, Li J, Yin YP, Wang B, Cohen MS, et al. HIV incidence among men who have sex with men in China: a meta-analysis of published studies. PLoS One. 2011;6:e23431

5. Berry M, Wirtz AL, Janayeva A, Ragoza V, Terlikbayeva A, Amirov B, et al. Risk factors for HIV and unprotected anal intercourse among men who have sex with men (MSM) in Almaty, Kazakhstan. PloS one. 2012;7:e43071.

6. Chow EP, Chen X, Zhao J, Zhuang X, Jing J, Zhang L. Factors associated with self-reported unprotected anal intercourse among men who have sex with men in Changsha city of Hunan province, China. AIDS Care. 2015;27:1332-42.

7. Yang Z, Zhang S, Dong Z, Jin M, Han J. Prevalence of unprotected anal intercourse in men who have sex with men recruited online versus offline: a meta-analysis. BMC Public Health. 2014;14:508.

8. Zhou Y, Li D, Lu D, Ruan Y, Qi X, Gao G. Prevalence of HIV and syphilis infection among men who have sex with men in China: a meta-analysis. Biomed Res Int. 2014;2014:620431.

9. Wu J, Hu Y, Jia Y, Su Y, Cui H, Liu H, et al. Prevalence of unprotected anal intercourse among men who have sex with men in China: an updated meta-analysis. PLoS One. 2014;9:e98366.

10. Chinese Center for Disease Control and Prevention Report. HIV/AIDS Epidemic in China. 2015.

11. Jie W, Ciyong L, Xueqing D, Hui W, Lingyao H. A syndemic of psychosocial problems places the MSM (men who have sex with men) population at greater risk of HIV infection. PLoS One. 2012;7:e32312.

12. Yu F, Nehl EJ, Zheng T, He N, Berg CJ, Lemieux AF, et al. A syndemic including cigarette smoking and sexual risk behaviors among a sample of MSM in Shanghai, China. Drug Alcohol Depend. 2013;132:265-70.
13. Wim VB, Christiana N, Marie L. Syndemic and other risk factors for unprotected anal intercourse among an online sample of Belgian HIV negative men who have sex with men. AIDS Behav. 2014;18:50-8.

14. Diaz RM, Ayala G, Bein E, Henne J, Marin BV. The impact of homophobia, poverty, and racism on the mental health of gay and bisexual Latino men: findings from 3 US cities. Am J Public Health. 2001;91:927-32.

15. Mimiaga MJ, O'Cleirigh C, Biello KB, Robertson AM, Safren SA, Coates TJ, et al. The effect of psychosocial syndemic production on 4-year HIV incidence and risk behavior in a large cohort of sexually active men who have sex with men. J Acquir Immune Defic Syndr. 2015;68:329-36.

16. Guadamuz TE, McCarthy K, Wimonsate W, Thienkrua W, Varangrat A, Chaikummao $S$, et al. Psychosocial health conditions and HIV prevalence and incidence in a cohort of men who have sex with men in Bangkok, Thailand: evidence of a syndemic effect. AIDS Behav. 2014;18:2089-96.

17. Mimiaga MJ, Noonan E, Donnell D, Safren SA, Koenen KC, Gortmaker S, et al. Childhood sexual abuse is highly associated with HIV risk-taking behavior and infection among MSM in the EXPLORE Study. J Acquir Immune Defic Syndr. 2009;51:340-8.

18. De Santis JP, Colin JM, Provencio Vasquez E, McCain GC. The relationship of depressive symptoms, self-esteem, and sexual behaviors in a predominantly Hispanic sample of men who have sex with men. Am J Mens Health. 2008;2:314-21.

19. Wilson PA, Stadler G, Boone MR, Bolger N. Fluctuations in depression and well-being are associated with sexual risk episodes among HIV-positive men. Health Psychol. 2014;33:681-5.

20. Preston DB, D'Augelli AR, Kassab CD, Cain RE, Schulze FW, Starks MT. The influence of stigma on the sexual risk behavior of rural men who have sex with men. AIDS Educ Prev. 2004;16:291-303.

21. Halkitis PN, Kupprat SA, Hampton MB, Perez-Figueroa R, Kingdon M, Eddy $J A$, et al. Evidence for a Syndemic in Aging HIV-positive Gay, Bisexual, and Other MSM: Implications for a Holistic Approach to Prevention and Healthcare. Nat Resour Model. 2012:36(2). doi:10.1111/napa.12009.

22. Ye J, Shim R, Rust G. Health care avoidance among people with serious psychological distress: analyses of 2007 Health Information National Trends Survey. J Health Care Poor Underserved. 2012;23:1620-9.

23. Zhou ZH, Li SM, Liu YJ, Jiang SL, Zhang XX, Li QC, et al. [Study on the relationship between behavioral factors, psychological status and HIV infection among men who have sex with men in Beijing.]. Zhonghua Liu Xing Bing Xue Za Zhi. 2010;31:273-6.

24. Parsons JT, Grov C, Golub SA. Sexual compulsivity, co-occurring psychosocial health problems, and HIV risk among gay and bisexual men: further evidence of a syndemic. Am J Public Health. 2012;102:156-62.

25. Operario D, Nemoto T. HIV in transgender communities: syndemic dynamics and a need for multicomponent interventions. J Acquir Immune Defic Syndr. 2010;55 Suppl 2:S91-3.

26. Stall R, Mills TC, Williamson J, Hart T, Greenwood G, Paul J, et al. Association of co-occurring psychosocial health problems and increased vulnerability to HIV/AIDS among urban men who have sex with men. Am J Public Health. 2003:93:939-42.

27. O'Leary D. The syndemic of AIDS and STDS among MSM. Linacre Q. 2014;81:12-37.

28. Singer M, Clair S. Syndemics and public health: reconceptualizing disease in bio-social context. Med Anthropol Q. 2003:17:423-41.

29. Center for Disease Control and prevention. Milstein B. Introduction to the Syndemics Prevention Network. Atlanta: Centers for Disease Control and Prevention; 2002. Available: https:/www.cdc.gov/nchhstp/ programintegration/Definitions.htm. Accessed 16 Dec 2016.

30. Meyer JP, Springer SA, Altice FL. Substance abuse, violence, and HIV in women: a literature review of the syndemic. J Women's Health. 2011;20: 991-1006.

31. Mustanski B, Garofalo R, Herrick A, Donenberg G. Psychosocial health problems increase risk for HIV among urban young men who have sex with men: preliminary evidence of a syndemic in need of attention. Ann Behav Med. 2007:34:37-45.

32. Santos GM, Do T, Beck J, Makofane K, Arreola S, Pyun T, et al. Syndemic conditions associated with increased HIV risk in a global sample of men who have sex with men. Sex Transm Infect. 2014;90:250-3.

33. Deuba K, Ekstrom AM, Shrestha R, lonita G, Bhatta L, Karki DK. Psychosocial health problems associated with increased HIV risk behavior among men who have sex with men in Nepal: a crosssectional survey. PLoS One. 2013;8:e58099. 
34. Lin H, Ding Y, Liu X, Wu Q, Shen W, He N. High prevalence of HIV infection and bisexual networks among a sample of men who have sex with men in eastern china. PLoS One. 2015;10:e0129300.

35. Chen X, Li X, Zheng J, Zhao J, He J, Zhang G, et al. Club drugs and HIV/STD infection: an exploratory analysis among men who have sex with men in Changsha, China. PloS one. 2015;10:e0126320.

36. Chen H, Li Y, Wang L, Zhang B. Causes of suicidal behaviors in men who have sex with men in China: a national questionnaire survey. BMC Public Health. 2015;15:91.

37. Li D, Li C, Wang Z, Lau JT. Prevalence and associated factors of unprotected anal intercourse with regular male sex partners among HIV negative men who have sex with men in China: a cross-sectional survey. PLoS One. 2015;10:e0119977.

38. Dyer TP, Shoptaw S, Guadamuz TE, Plankey M, Kao U, Ostrow D, et al. Application of syndemic theory to black men who have sex with men in the multicenter AIDS cohort study. J Urban Health. 2012;89:697-708.

39. Mimiaga MJ, Biello KB, Robertson AM, Oldenburg CE, Rosenberger JG, O'Cleirigh C, et al. High prevalence of multiple syndemic conditions associated with sexual risk behavior and HIV infection among a large sample of Spanish- and Portuguese-speaking men who have sex with men in Latin America. Arch Sex Behav. 2015;44:1869-78.

40. le Giang M, Viet VD, Hao BT. Sexual health and men who have sex with men in Vietnam: an integrated approach to preventive health care. Adv Prev Med. 2012;2012:796192.

41. Herrick AL, Lim SH, Plankey MW, Chmiel JS, Guadamuz TE, Kao U, et al. Adversity and syndemic production among men participating in the multicenter AIDS cohort study: a life-course approach. Am J Public Health. 2013;103:79-85.

42. Cantwell J, Muldoon O, Gallagher S. The influence of self-esteem and social support on the relationship between stigma and depressive symptomology in parents caring for children with intellectual disabilities. J Intellect Disabil Res. 2015;59:948-57.

43. Tucker A, Liht J, de Swardt G, Jobson G, Rebe K, Mclntyre J, et al. Homophobic stigma, depression, self-efficacy and unprotected anal intercourse for peri-urban township men who have sex with men in Cape Town, South Africa: a cross-sectional association model. AIDS Care. 2014:26:882-9.

44. Hubach RD, Dodge B, Li MJ, Schick V, Herbenick D, Ramos WD, et al. Loneliness, HIV-related stigma, and condom use among a predominantly rural sample of HIV-positive men who have sex with men (MSM). AIDS Educ Prev. 2015;27:72-83.

45. Taylor SE, Brown JD. Illusion and well-being: a social psychological perspective on mental health. Psychol Bull. 1988;103:193-210.

46. Robins RW, Hendin HM, Trzesniewski KH. Measuring Global Self-Esteem: Construct Validation of a Single-Item Measure and the Rosenberg SelfEsteem Scale[J]. Person Soc Psychol Bull. 2001;27(2):151-61.

47. Rosenberg M. Society and the adolescent self-image. Princeton: Princeton University Press; 1965.

48. Swinson R. The GAD-7 scale was accurate for diagnosing generalized anxiety disorder. Evid Based Med. 2006;11(6):184.

49. Ferlatte O, Hottes TS, Trussler T, Marchand R. Evidence of a syndemic among young Canadian gay and bisexual men: uncovering the associations between anti-gay experiences, psychosocial issues, and HIV risk. AIDS Behav. 2014;18:1256-63.

\section{Submit your next manuscript to BioMed Central and we will help you at every step:}

- We accept pre-submission inquiries

- Our selector tool helps you to find the most relevant journal

- We provide round the clock customer support

- Convenient online submission

- Thorough peer review

- Inclusion in PubMed and all major indexing services

- Maximum visibility for your research

Submit your manuscript at www.biomedcentral.com/submit

C Biomed Central 